\title{
Mapping of emerging territorial phenomena at Micro Scale: Development of collaborative database as a base for Evidence-Based Design Strategies
}

\author{
Habid Becerra-Santacruz \\ Universidad Michoacana de San Nicolas de Hidalgo | México | hbecerra@umich.mx
}

Axel Becerra-Santacruz

Universidad Michoacana de San Nicolas de Hidalgo | México | axel.becerra@umich.mx

\begin{abstract}
This paper presents an active analysis and research approach for design workshops at the Faculty of Architecture at UMNSH. The proposed scheme for final year design studio demands students to participate in the confrontation of reality to understand first-hand through databases; the complex problems of contemporary society and its relationship with the habitat. In order to understand the diverse emergent phenomena of the city, a collaborative work is implemented for the development of a database, occupation maps and territorial dynamics on a micro scale. From the evidence supported by data, students articulate design strategies and specific territorial actions.
\end{abstract}

Keywords: Collaborative database, evidence-based design strategies, emergent phenomena mapping, design pedagogy.

\section{INTRODUCCIÓN}

Los talleres en la enseñanza de la arquitectura y diseño urbano enfrentan diversos retos y desafíos relacionados con los cambios y dinámicas de una sociedad contemporánea y su hábitat.

Dentro de los talleres de fin de carrera, comúnmente se desarrolla una investigación donde se identifica la problemática de un usuario o comunidad, para la cual se plantean soluciones a través de directrices de diseño convencional. Como resultado, a través de esta metodología, es muy común que los alumnos en conjunto con sus profesores definan especulativamente un programa arquitectónico en base a necesidades identificadas. Posteriormente se sigue el desarrollo del trabajo con estas bases hasta llegar a un proyecto en sus diversas fases de carácter ejecutivo.

Lamentablemente este proceso cae en la trampa de autovalidación académica, en donde se discute y evalúa principalmente entre la misma comunidad de profesores y en algunas ocasiones con arquitectos invitados, sin el escrutinio de las contingencias cotidianas de la sociedad y su realidad inmediata. (Till, 2009)

El presente artículo plantea como primer paso para confrontar la realidad inmediata; un esquema de trabajo basado en construcción de bases de datos a través acción colectiva, (acto u operación que implica actividad, movimiento o cambio) a través de un grupo de alumnos agentes y profesores que actúan voluntariamente, en oposición a quietud. Posteriormente se analizan los datos para articular estrategias de diseño, así como acciones de territoriales específicas.
Este modelo pedagógico también llamado aprendizaje basado en proyectos activos, ha logrado vincular a los alumnos de diversos programas a nivel licenciatura y posgrado, (alumnos de Licenciatura en Arquitectura (LIC), Especialidad en Restauración de Sitios y Monumentos (ERSM) y de la Maestría en Diseño Avanzado (MDA) de la Facultad de Arquitectura de la Universidad Michoacana de San Nicolás de Hidalgo), con diversas comunidades, sus ayuntamientos, instituciones públicas y asociaciones civiles.

\section{METODOLOGÍA}

El proceso metodológico contempla, recolección de datos, análisis de datos, visualización de datos, diagnóstico, planteamiento estratégico, diseño, gestión e implementación de proyectos a pequeña escala (Figura 1).

La recolección de información inicia desde entender con datos duros la realidad directamente en el sitio de estudio a través de diversas herramientas e instrumentos tecnológicos. El diagnóstico basado en evidencia permite plantear proyectos estratégicos que impacten directamente a las problemáticas prioritarias de la comunidad de estudio. Esto permite a los estudiantes conocer diferentes variables territoriales que presentan la ciudad y así contrastar estas características con el resto del territorio a través de diversas dimensiones tales como: accesibilidad, medio ambiente, infraestructura y aspectos socioeconómicos, etc. El objetivo principal es entender a la ciudad y su sociedad como un ente dinámico y en constante movimiento. Castells (1998) define a los flujos como la nueva materia de la que nuestras sociedades y ciudades están hechas. 


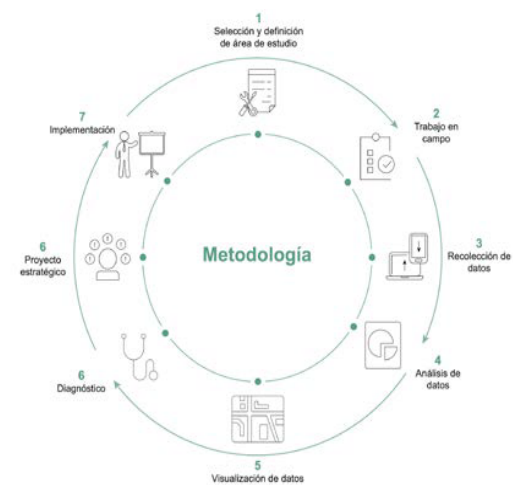

Figura 1: Diagrama metodológico aplicado. Fuente: Acervo de autores

\section{DELIMITACIÓN DE LA ZONA DE ESTUDIO TRABAJO DE CAMPO}

La metodología expuesta en este artículo ha sido aplicada en diversos estudios específicos destacando el área geográfica del municipio de Morelia (Tabla 1).

Dentro del taller de diseño se definen los criterios para la selección y delimitación de área de estudio y definición de objetivos particulares, en base a condiciones de segregación, grado de marginación, déficit de servicios, infraestructura o equipamiento. La delimitación de la zona de estudio a su vez es fuertemente influenciada por la capacidad de recursos disponibles, resaltando que dentro de la metodología expuesta se registra el 100 de territorio a escala predial y aplicación de encuestas de percepción con una representatividad del 7 al $10 \%$.

\section{RECOLECCION DE DATOS}

La recolección de información se convierte el elemento medular del taller de diseño, los participantes confrontan la realidad directamente en campo mediante el apoyo de diversas herramientas e instrumentos tecnológicos.

Previo al inicio de recolección de datos se definen las herramientas a utilizar en base a las características particulares de la zona de estudio, determinado por las características de un contexto urbano, rur-urbano o rural, si tiene fácil accesibilidad, cobertura de red de datos, etc. Las herramientas de recolección para registro de uso de suelo, fenómenos territoriales a escala predial y aplicación de encuestas de percepción se realiza mediante uso aplicaciones móviles que de manera simultanea georreferencian los datos obtenidos. Al mismo tiempo se registran levantamientos de imágenes aéreas mediante el uso de drones.
Una etapa de gran relevancia es la capacitación, definición de criterios y variables para la recolección de datos con el objetivo de homogenizar y estandarizar la información. Lo anterior se lleva a cabo mediante talleres plenarios (con todos los participantes, los talleres de capacitación concluyen con una prueba piloto aplicando todos los instrumentos para la recolección de información (Figura 2).

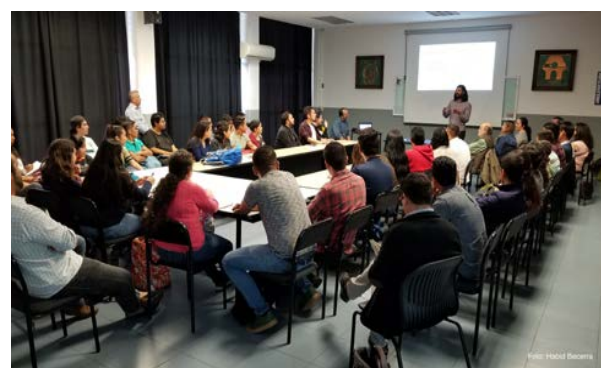

Figura 2: Talleres plenarios de capacitación para alumnos. Fuente: Acervo de autores

\section{REGISTRO DE FENÓMENOS TERRITORIALES A ESCALA PREDIAL}

Dentro de este apartado se registran variables y fenómenos territoriales que ayuden a tener una lectura de primer mano y de manera paralela se actualice o construya una base de datos colaborativa para definición de diseño basado en evidencia. Las variables que se registran en el territorio a escala predial contemplan las categorías de uso de suelo, tipología de vivienda, equipamiento urbano, niveles de construcción, estado de conservación, servicios, infraestructura. El numero de elementos recolectados por predio oscila entre 15 y 22 variables que de manera simultánea incorpora su georefencia.

\section{ENCUESTA DE PERCEPCIÓN.}

La encuesta de percepción es aplicada cubriendo todo el territorio en estudio buscando una representatividad estadística en el orden del 7 al 10\% (Figura 3). La encuesta en términos generales recolecta datos básicos de identificación, genero, edad, nivel educativo, rol dentro la familia, nivel socioeconómico, esquema de movilidad, consultas sobre nivel de percepción y satisfacción con relación a los servicios, equipamiento, espacio público, la seguridad etc. De igual manera se incorpora una sección con preguntas para identificar fenómenos en la vida barrial, niveles de participación ciudadana, carencias del sitio, lugares de conflicto, principales problemáticas, etc.

\section{El diseño del instrumento se conforma de 3 categorías:}

- Datos de necesidades básicas y lugares conflictivos. Preguntas abiertas

Tabla 1: Datos de los estudios realizados bajo la metodología expuesta. Elaboración propia.

\begin{tabular}{|c|c|c|c|c|c|}
\hline Área de estudio & & $\begin{array}{c}\text { Superficie en } \\
\mathrm{Km}^{2}\end{array}$ & Predios registrados & $\begin{array}{c}\text { Encuestas de } \\
\text { percepción }\end{array}$ & $\begin{array}{c}\text { Alumnos } \\
\text { participantes }\end{array}$ \\
\hline $\begin{array}{l}\text { Villas del Pedregal, } \\
\text { Michoacán }\end{array}$ & Morelia, & 1.95 & 10161 & 539 & 18 \\
\hline Lagunillas, Michoacán & & 1.98 & 1357 & 68 & 12 \\
\hline $\begin{array}{l}\text { Centro Histórico, } \\
\text { Michoacán }\end{array}$ & Morelia, & 3.38 & 7035 & 665 & 40 \\
\hline
\end{tabular}


- Datos de valoración, percepción al contexto, actores y servicios. Escala Likert

- Datos socioeconómicos. Escala de valoración
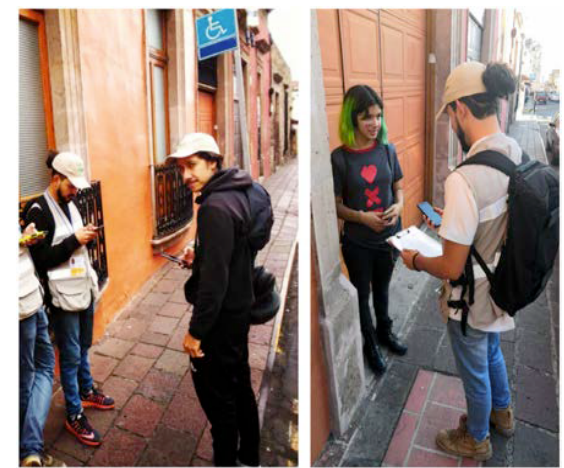

Figura 3: Levantamiento en campo y aplicación de encuestas Fuente: Acervo de autores

\section{HERRAMIENTAS}

Hoy en día existen gran variedad de herramientas disponibles para recolectar datos en campo y generar bases de datos, algunas de ellas fueron diseñadas originalmente para generar grandes cantidades de información para mejorar servicios de transporte público (Ching, Zegras, Kennedy, \& Mamun, 2012). Sin duda alguna, la elección de herramientas adecuadas es quizá una de las tareas mas importantes que determinan la eficiencia y eficacia, así como aprovechamiento de recursos para la construcción colaborativa de plataformas como es el objeto de estudio en el presente articulo.

De los cuatro estudios citados anteriormente en la sección delimitación del área de estudio, se registraron tres proyectos. Todos los datos se levantaron durante el punto exacto, mediante la aplicación móvil Flocktracker@ para dispositivos Android (MIT Department of Urban Studies, and Planning, Mobility Futures Collaborative, and Urban Launchpad/MX, 2016), la cual fue desarrollada por el Instituto Tecnológico de Massachusets MIT (Figura 4). Esta aplicación se ha sido utilizada previamente para mapear rutas de tránsito informales y aplicar encuestas a bordo de pasajeros en Dhaka, Ciudad de México y Solo (Zegras et al., 2015b, a; Kota Kita, 2015). Flocktracker@ permite diseñar de manera flexible formularios que permiten registrar un sin numero de variables a los cuales permite incorporar georeferencia y anexos fotográficos si el estudio lo requiere (Figura 5).
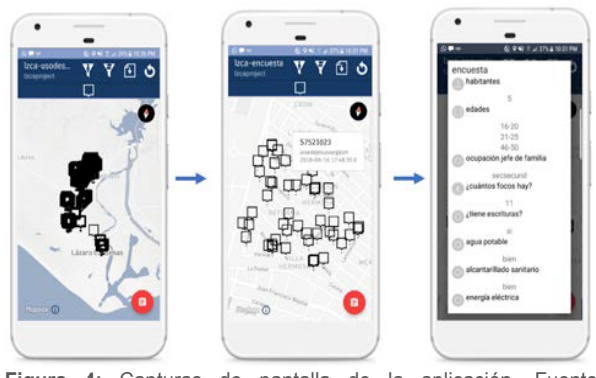

Figura 4: Capturas de pantalla de la aplicación. Fuente Flocktracker@

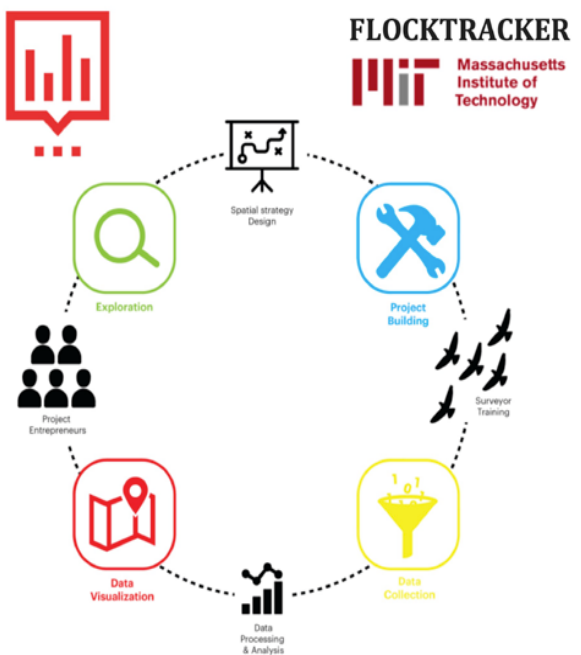

Figura 5: Flujo de trabajo de aplicación. Fuente Flocktracker

Durante el levantamiento permite a los administradores observar en tiempo real el territorio cubierto y visualizar las diversas variables registradas. Los datos son transmitidos mediante red móvil a un servidor en la nube para su futuro análisis y procesamiento (Figura 6).

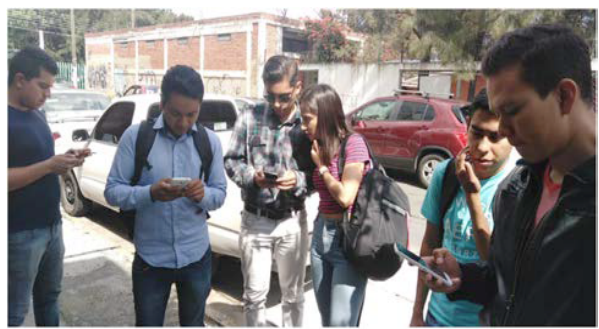

Figura 6: Levantamiento en campo mediante uso de aplicaciones móvil. Fuente: Acervo de autores

Es importante señalar que el más reciente estudio desarrollado en el Centro Histórico de Morelia por el Instituto Municipal de Planeación Morelia IMPLAN, tomo las experiencias metodológicas previas para desarrollar su propia aplicación para recolección de datos MappMorelia@ (IMPLAN Instituto Municipal de Planeación Morelia, 2019) (Figura 7).
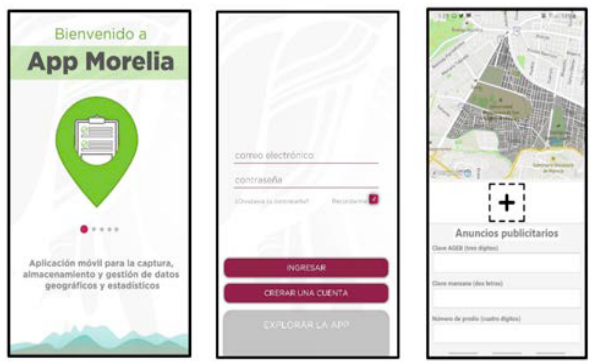

Figura 7: Capturas de pantalla de la aplicación. Fuente MappMorelia@ 
Dicha aplicación oferta herramientas similares a la aplicación Flocktracker@, sin embargo, se enriquece incorporando una plataforma web la cual permite registros desde cualquier dispositivo iOS y Android. De igual forma permite cargar capas y planos base del territorio en estudio de acuerdo a las necesidades particulares. Los datos son registrados en tiempo real de igual forma que la aplicación Flocktracker@

\section{BASE DE DATOS}

El registro de datos en tiempo real mediante aplicaciones móvil, ha permitido la construcción de base de datos colaborativa como se ha descrito anteriormente. Ambas aplicaciones Flocktracker@ y MappMorelia@ generan hojas de cálculo con registros individuales de acuerdo al diseño de cada formulario. La construcción de base de datos se desarrolla en tiempo real, la cual es alimentada de manera colaborativo por todos los estudiantes, posteriormente la información es analizada y procesada.

Como parte de la logística para el levantamiento de datos y creación de base de datos es importante definir las rutas de cobertura de cada uno de los participantes y al mismo tiempo establecer una nomenclatura que permita construir una clave de registro único por predio. La clave de identificación única por predio es compuesta por el id del área geográfica regional (AGEB), el id de la manzana y el número de predio dentro de la manzana (Figura 8).
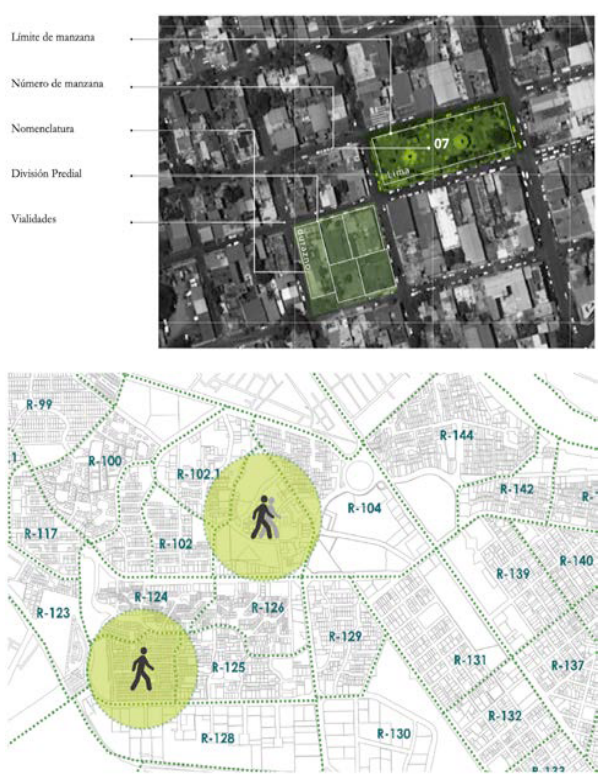

Figura 8: Nomenclatura y rutas para levantamiento de datos. Fuente: Acervo de autores

\section{ANALISIS Y VIZUALIZACION DE DATOS}

Dentro de esta etapa se generan insumos y productos que se convierten en las herramientas fundamentales para establecer un diagnóstico el cual informará directamente el diseño basado en la evidencia recolectada en sitio. La información recolectada a nivel predial es analizada y procesada para posteriormente desarrollar mapeos de los diversos fenómenos territoriales (Figura 9).

En cuanto a la encuesta de percepción también es analizada y procesada resaltando los fenómenos representativos en el territorio generando gráficas con las cuales se identifican, desde la apreciación de los residentes, las principales carencias y problemáticas del sitio (Figura 10).

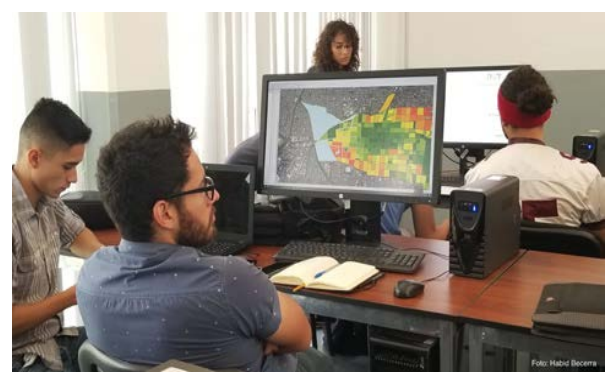

Figura 9: Capacitación de herramientas GIS y desarrollo de mapeos. Fuente: Acervo de autores
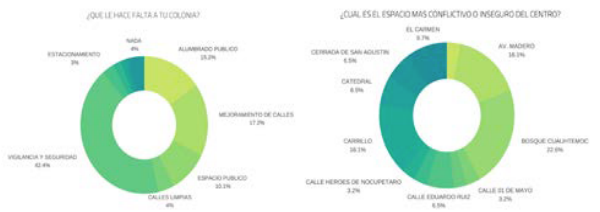

Figura 10: Procesamiento y visualización de encuestas de percepción. Elaboración propia

\section{DIAGNÓSTICO}

Es importante resaltar que el objetivo del esquema de trabajo en discusión es construir un diagnostico basado en evidencias que permita identificar problemas e identificar las zonas físicas vulnerables a atender, analizando aspectos paisajísticos, socioeconómicos, de equipamiento, infraestructura y uso de suelo.

Para definir el mismo se recurre a otro instrumento importante a través de talleres participativos, los cuales toman parte en diversas etapas del estudio. Los talleres participativos tienen como objetivo resaltar las necesidades y problemáticas comunes de los habitantes del territorio en estudio, teniendo dicha información de primera mano incorporando grupos representativos de cada sector (Figura 11)

Los talleres participativos en su primera fase están diseñados bajo la siguiente estructura:

- Construcción de línea de tiempo: recopila información relevante sobre la historia del lugar, sus etapas de construcción conflictos y eventos trascendentales para la identidad y el estado actual

- Cartografía social: Se hace un reconocimiento del territorio mediante la representación grafica en planta del mismo. Se identifican los lugares mas concurridos, los menos usados, rutas, zonas de riesgo, etc.

- Actores: Se identifican personas, instituciones, organizaciones sin fines de lucro, sociedades y asociaciones civiles, etc. $Y$ se categorizan en tres 
grupos: actores negativos, neutrales y positivos. Esto permite crear una ruta de acción y gestión para los proyectos propuestos.

- Problemas y soluciones: Mediante un diagrama de árbol se identifican las problemáticas del territorio más urgentes y se proponen soluciones.

- Priorización: Este es un ejercicio que busca decidir cual o cuales de las problemáticas planteadas representan un problema de atención prioritaria.
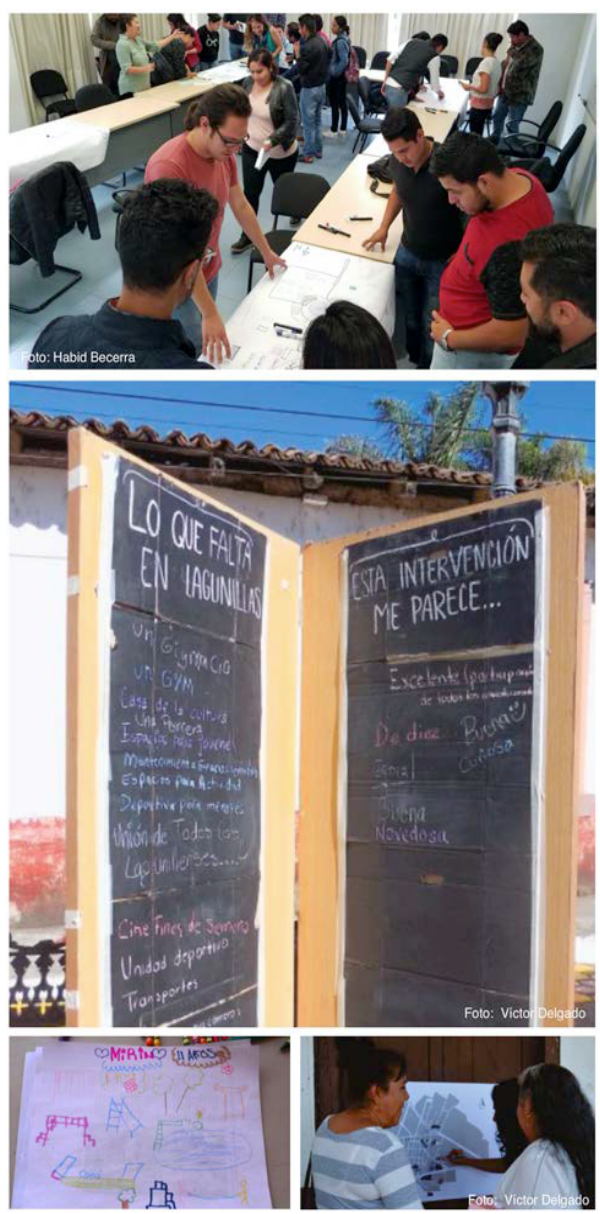

Figura 11: Talleres participativos, cartografía social, actores, priorización de problemáticas y soluciones. Fuente: Acervo de autores

\section{PLAN MAESTRO Y PROYECTOS ESTRATÉGICOS}

Derivado de la información encuestada, registros territoriales a escala predial, los diagnósticos realizados y del análisis resultado del ejercicio analítico acerca de la situación general de la comunidad de estudio, los participantes del taller definen las líneas y ejes estratégicos que incidirán en las problemáticas detectadas en sitio desarrollando un plan de acción y plan maestro.

El plan maestro se consolida a través de un proceso que atiende áreas de acción prioritaria identificadas por el trabajo de campo y obtención de datos basados en evidencia. Dentro del plan maestro a su vez se define la implementación de estrategias a corto mediano y largo plazo acompañado de proyectos específicos. Lo anterior con el objetivo de crear contextos propicios para el desarrollo de ambientes prósperos, incluyentes y equitativos construidos desde una visión ciudadana. (SEDATU 2020).

Los proyectos estratégicos son desarrollados posteriormente por los alumnos de manera individual quienes informan el proceso de diseño a partir de la evidencia (Figura 12). El ejercicio académico concluye con un documento de tesis, el cual debe ser defendido por el alumno sustentante.
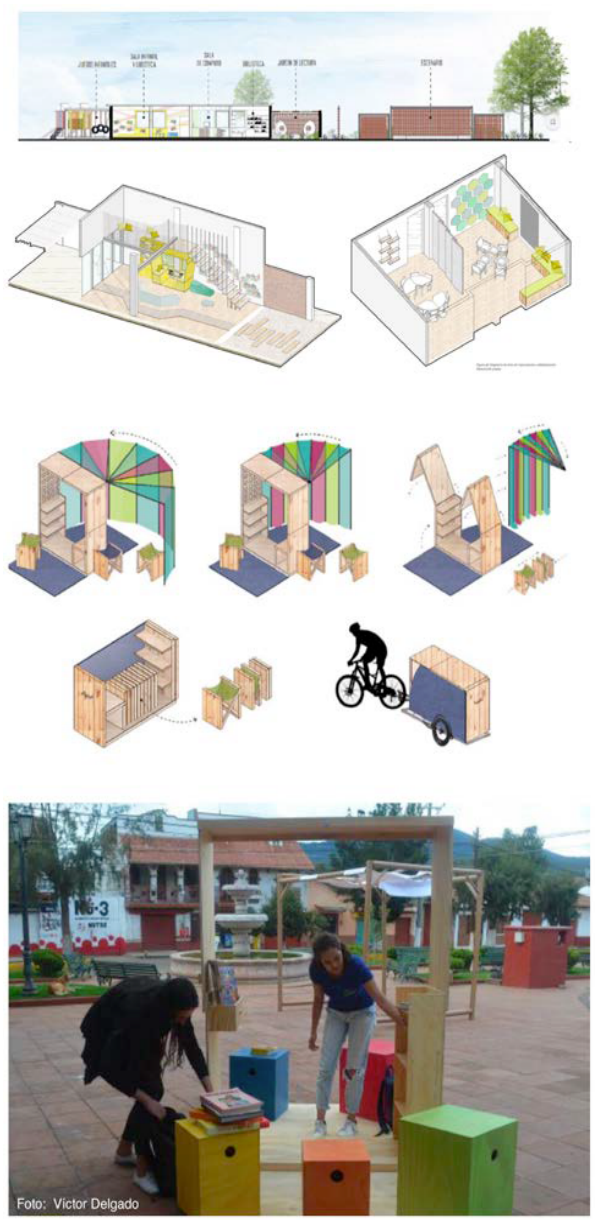

Figura 12: Ejemplo de proyecto estratégico para el municipio de Lagunillas, Michoacán. Proyecto Parque del Libro con extensión de módulo itinerante "libro rodante". Autor: Larisa Calderón

\section{INTERVENCIÓN}

Se destaca dentro del esquema de trabajo, el Taller de Acción Rur-Urbana (TARU), el cual hace énfasis en formar diseñadores proactivos que actúen voluntariamente en el territorio mediante procesos de innovación social que concentran acciones livianas, rápidas y baratas llamadas tácticas urbanas, estrategias que han sido impulsadas en diversos países latinoamericanos a través del laboratorio de Ciudad Emergente (2019). Informados por la 
retroalimentación de los talleres participativos, los alumnos seleccionan una pequeña intervención, la cual es desarrollada de forma emergente para evaluar el impacto que puede tener el diseño a través de la acción colectiva (Figura 13).
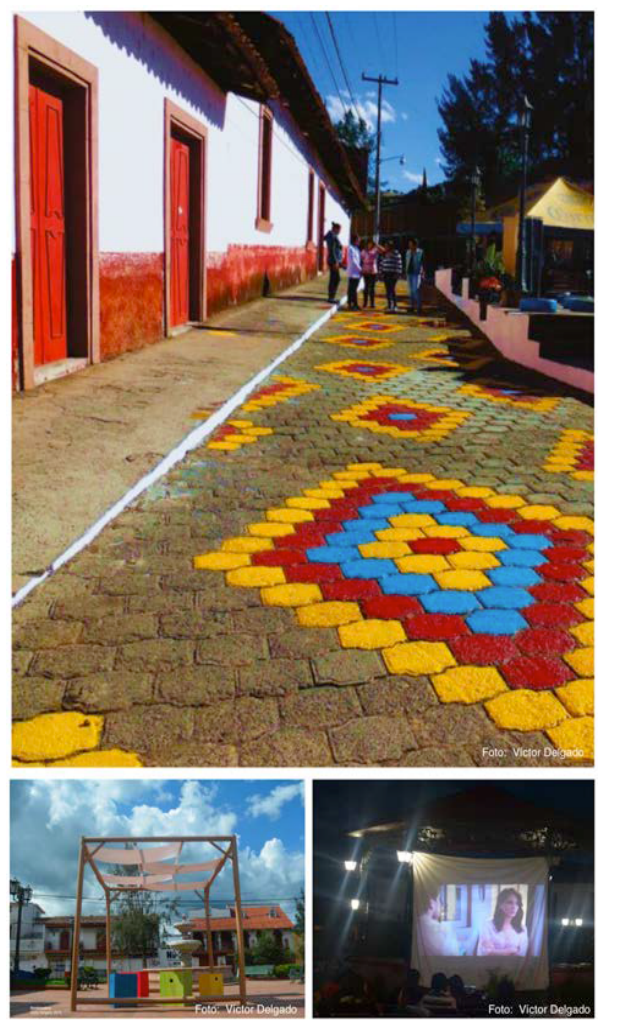

Figura 13: Intervenciones en Lagunillas, implementación de senderos, sombras en espacio público y cinema itinerante. Fuente: Acervo de autores

\section{CONCLUSIONES}

La aplicación de este esquema metodológico en talleres de Diseño de la Facultad de Arquitectura de la Universidad Michoacana de San Nicolás de Hidalgo, marca un referente como primera experiencia desarrollada de este carácter, el modelo pedagógico incide directamente en informar los procesos de enseñanza en la arquitectura desde una visión integral a partir de la evidencia soportada por datos duros.

El esquema de trabajo expuesto plantea acciones puntuales para una incentivar una dinámica social interactiva con los ciudadanos.

La generación de evidencia y en concreto el desarrollo de bases de datos, mapeos de fenómenos emergentes se convierten en el soporte para diseñar y planificar ciudades centradas en las personas mejorando la calidad de vida de sus habitantes. La toma de decisiones basada en evidencia permite identificar oportunidades y áreas potenciales de intervención que pueden ser transformados en políticas públicas, planes de desarrollo urbano y proyectos.

El presente artículo sienta las bases para el desarrollo de futuras investigaciones en las cuales deberá enfatizar puntualmente sobre los contrastes e impactos del modelo pedagógico expuesto. Al mismo tiempo se identifica la necesidad de desarrollar instrumentos e indicadores que permitan dimensionar puntualmente los fenómenos territoriales registrados y determinar su incidencia en la calidad de vida de los habitantes.

\section{AGRADECIMIENTOS}

Los autores expresan un profundo agradecimiento a todos los alumnos participantes en el presente trabajo. Al Instituto Municipal de Planeación Morelia IMPLAN por la colaboración y transferencia de conocimiento. A Daniel Heriberto Palencia por el apoyo y soporte para el uso de la aplicación Flocktracker.

\section{REFERENCIAS}

Castells, M., \& Blackwell, C. (1998). The information age: economy, society and culture. Volume 1 . The rise of the network society. Environment and Planning B: Planning and Design, 25, 631-636.

Ciudad Emergente. (2019). Retrieved from https://ciudademergente.org/

Ching, A., Zegras, C., Kennedy, S., \& Mamun, M. (2012). A UserFlocksourced Bus Experiment in Dhaka:New Data Collection Technique with Smartphones. Transportation Research Board Annual Meeting. Kota Kita, 2015. The Angkots of Solo.

IMPLAN Instituto Municipal de Planeación Morelia, 2019. "AppMorelia" Retrieved from https://implanapp.morelia.gob.mx/

Kota Kita, Solo. Retrieved from http://www.kotakita.org/projectangkot-study.html>.

MIT Department of Urban Studies, and Planning, Mobility Futures Collaborative, and Urban Launchpad/MX. 2016. "Flocktracker." Retrieved from http://www.flocktracker.org/

SEDATU 2020. Estrategia Nacional de Ordenamiento Territorial ENOT. Secretaría de Desarrollo Agrario, Territorial y Urbano.

Till, J., 2009. Architecture Depends. 1st ed. Cambridge, Mass: MIT Press, p.254.

Zegras, Christopher, Butts, Kuan, Cadena, Arturo, Palencia, Daniel, 2015a. Spatiotemporal dynamics in public transport personal security perceptions: digital evidence from Mexico City's periphery. In: Rietveld, Piet, Givoni, Moshe, Thomopoulos, Nikolas (Eds.), ICT for Transport: Opportunities and Threats. Edward ElgarPublishing, NY.

Zegras, Christopher, Eros, Emily, Butts, Kuan, Resor, Elizabeth, Kennedy, Stephen, Ching, Albert, Mamun, Muntasir, 2015b. Tracing a path to knowledge? Indicative user impacts of introducing a public transport map in Dhaka, Bangladesh." Cambridge Journal of Regions, Economy and Society 8.1 (2015): 113-129. 